\title{
Journal of Health Education and Health Promotion
}

\author{
www.journal.ihepsa.ir \\ Original Article
}

\section{The Survey of Factors Related to Consumption of Omega-3 Supple- ments Based on the Constructs of Health Belief Model in the Elderly Alzheimer Prevention}

\section{$\underline{\text { Zahra Jalili }}^{1}$, Reza Tavakoli ${ }^{1}$, Sahar Jalili ${ }^{2}$}

1. Associate Professor, Dept. of Health Education and Promotion, Science and Research Branch, Islamic Azad University, Tehran, Iran

2. Assistant Professor, Dept. of Fisheries, Abadan Branch, Islamic Azad University, Abadan, Iran

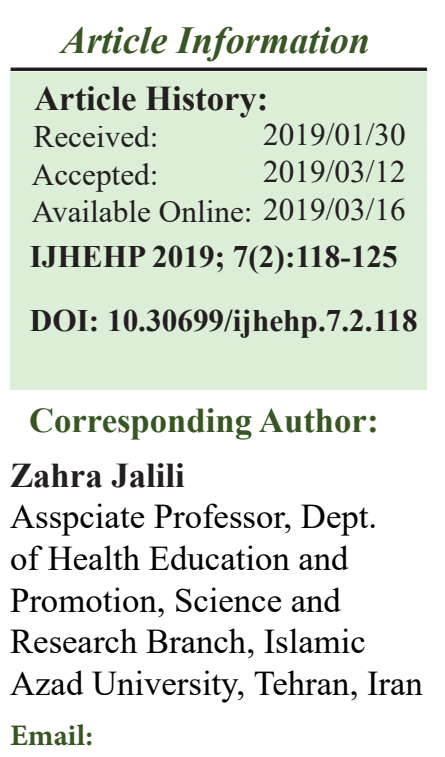

Zahra.Jalili@gmail.com

Use your device to scan and read the article online

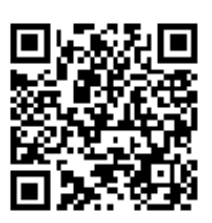

How to cite this article:

\begin{abstract}
Background and Objective: Threatening 5\%-10\% of people over 65 , Alzheimer's disease (AD) is the most common type of dementia. Studies recommended the consumption of substances containing omega-3 as a way to prevent $\mathrm{AD}$. The aim of present study was to determine factors related to consumption of omega-3 supplements based on the health belief model (HBM) constructs in order to prevent $\mathrm{AD}$ among the elderly.
\end{abstract}

Methods: In this analytical cross-sectional study, 333 elderly, who referred to the healthcare centers of Tehran city in 2015-2016, were selected by stratified random sampling method. To be over 60 years old, absence of AD and consumption of omega-3 supplements during the past year, were set as the study entry criteria. Data were collected by a questionnaire developed based on HBM constructs after determining its validity and reliability were determined. The collected data were analyzed using SPSS 16.

Results: The highest calculated mean belonged to the preventive behavior construct $48.6(P<0.001)$ followed by Perceived benefits $42.3(P<0.001)$. The lowest mean value was acquired by the construct of perceived barriers. This study showed that there existed some significant correlations of the behavior of omega-3 supplement consumption with the knowledge and constructs of susceptibility, severity and benefits perceived by the elderly studied.

Conclusion: Considering the present study results showing significant relationship of omega-3 supplement consumption with knowledge and majority of HBM constructs, it is suggested that some elderly education interventions be designed and implemented to promote the behavior of AD prevention through strengthening the constructs of HBM.

Keywords: Alzheimer's disease, Health belief model, Omega-3 supplementation, Elderly

Copyright (C) 2019 Journal of Health Education and Health Promotion. All rights reserved

Jalili Z, Tavakoli R, Jalili S. The Survey of Factors Related to Consumption of Omega-3 Supplements Based on the Constructs of Health Belief Model in the Elderly Alzheimer Prevention. Iran J Health Educ Health Promot. 2019; 7 (2) :118-125

Jalili, Z., Tavakoli, R., \& Jalili S. (2019) The Survey of Factors Related to Consumption of Omega-3 Supplements Based on the Constructs of Health Belief Model in the Elderly Alzheimer Prevention. Iranian Journal of Health Education and Health Promotion, 7 (2), 118-125

Iran J Health Educ Health Promot| فصلنامئ علمى يزوهشى آموزش بهداشت و ارتقاى سلامت 


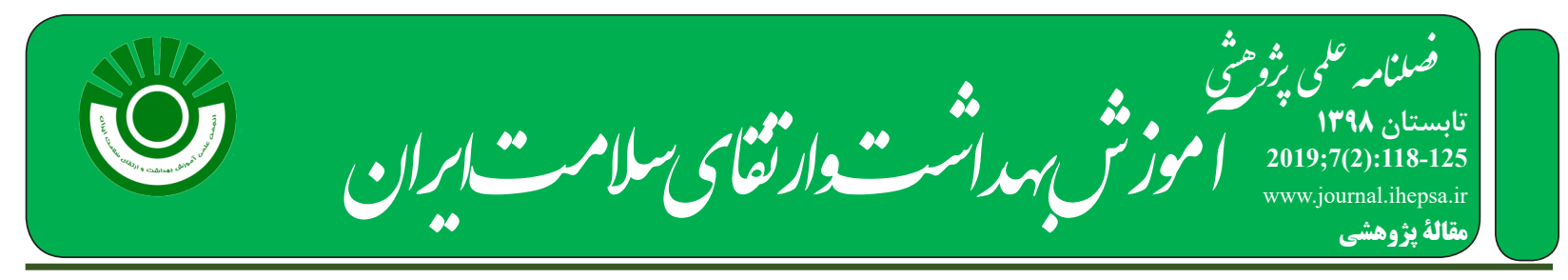

بررسى عوامل مرتبط با مصرف مكمل امعًا ب مبتنى بر سازههاى مدل اعتقاد بهـداشتى در وِيشَّيرى از بيمارى آلزايمر سالمندان

زهر اجليلى'، رضاتوكلى'، سحر جليلى'

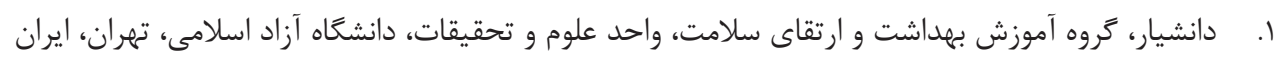

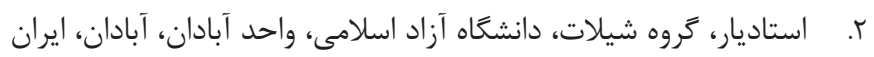

\begin{tabular}{|c|c|}
\hline جكيده & اطلاعات مقاله \\
\hline 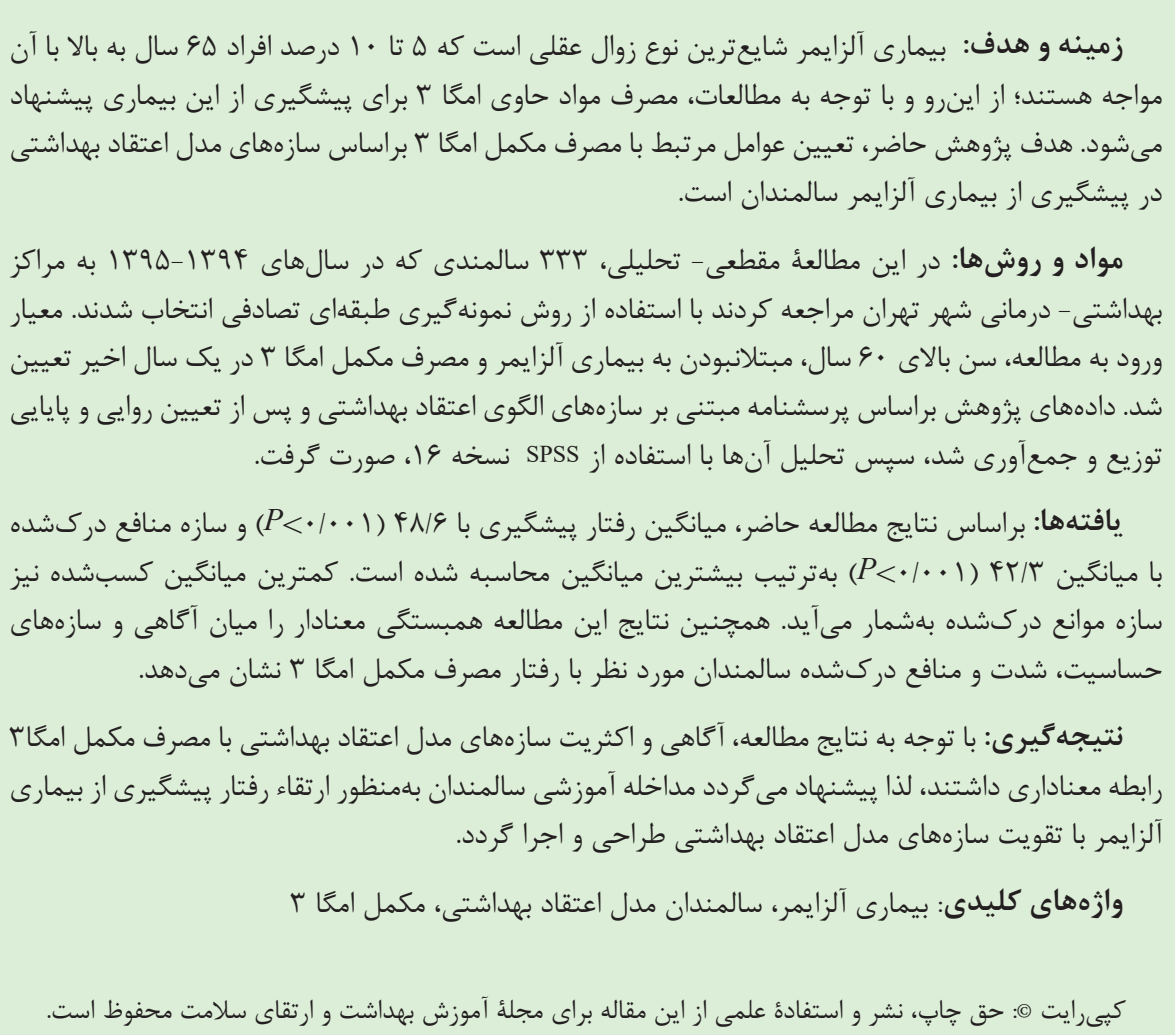 & 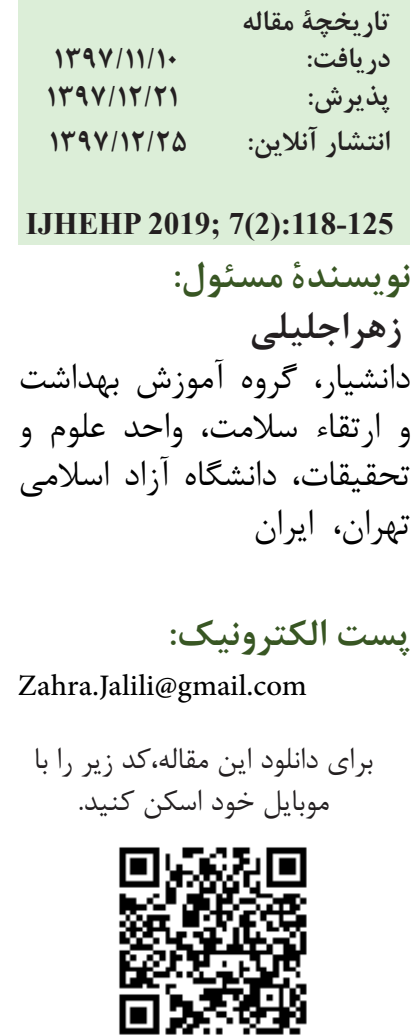 \\
\hline
\end{tabular}

از · لمه هزار نفر به اين بيمارى مبتلا هستند (9). بيمارى

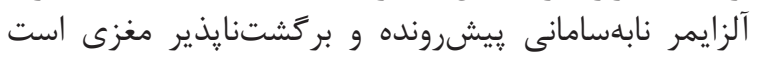

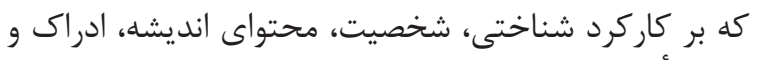

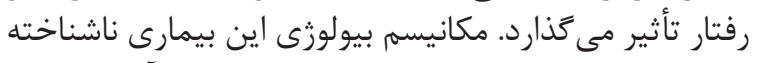

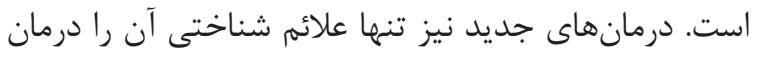

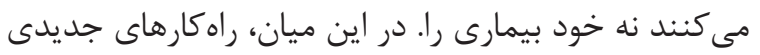

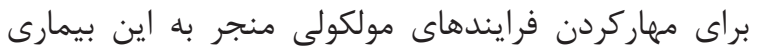

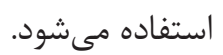

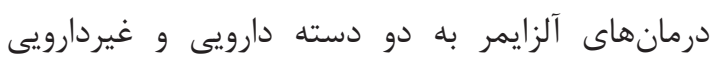

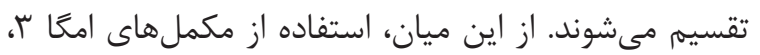

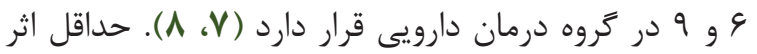

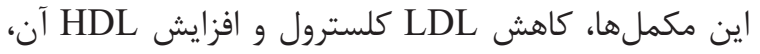
همجنين كمك به بهبود علائم بيمارى آلزايمر است. بسيارى

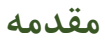

افزايش جمعيت سالمندان از ساير گروههاى سنى بـ بيشتر

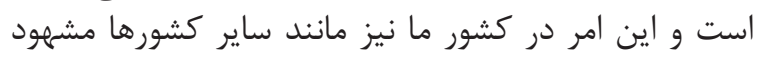

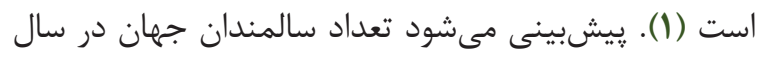

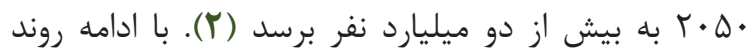

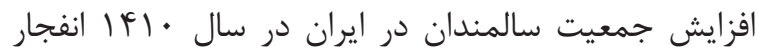

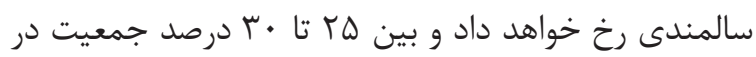

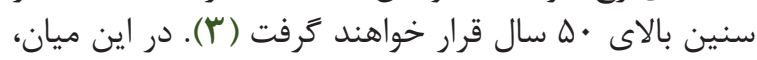

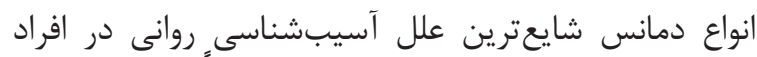

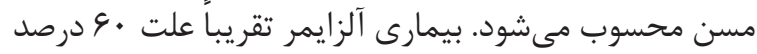

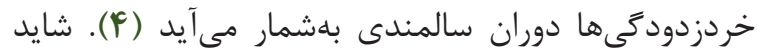

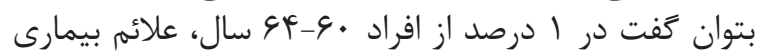

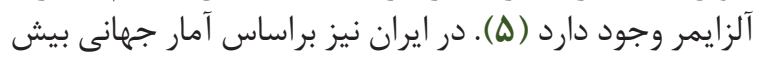


برنامهريزى آموزشى رفتارهاى ييشَّيرى از بيمارى آلزايمر براى برنامهريزان و متخصصان آموزش بهرئ بهداشت باشدي.

\section{مواد و روشها}

يزوهش حاضر مقطعى - تحليلى است و هدف مآ آن تعيين

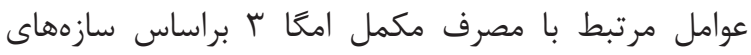

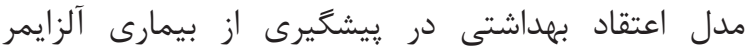

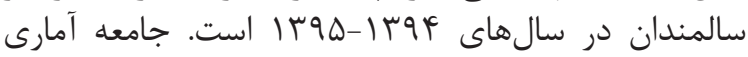

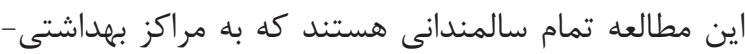

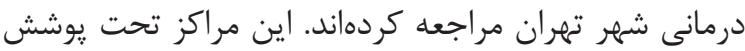

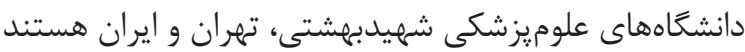

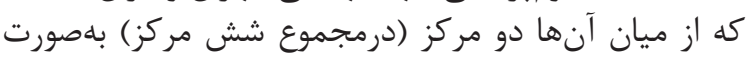

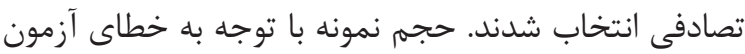
با

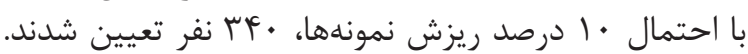

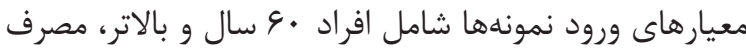

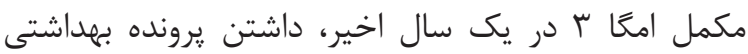

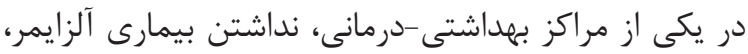

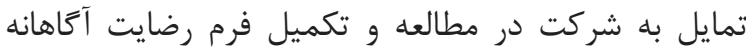

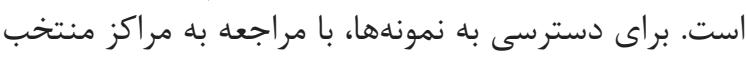

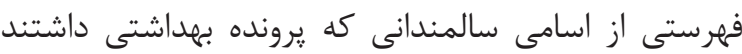

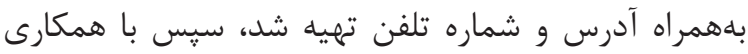

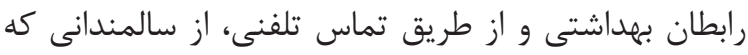

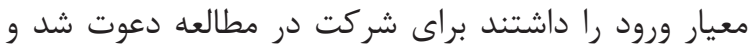

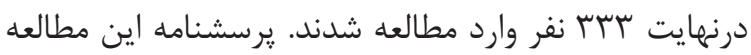

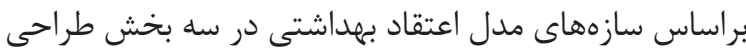

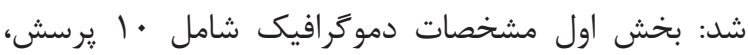

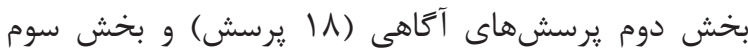

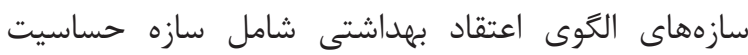

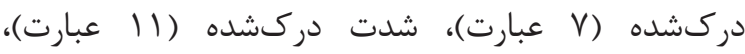

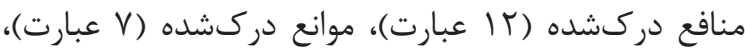

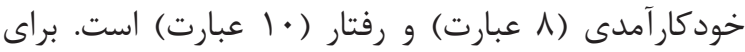

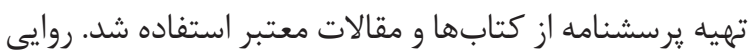

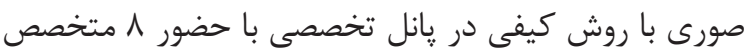

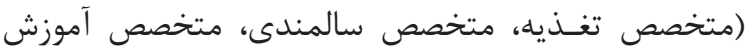

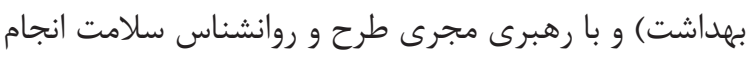

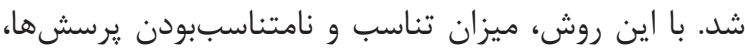

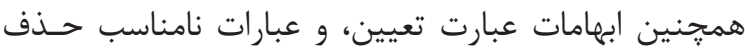

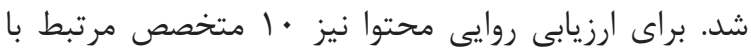

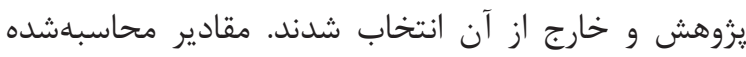

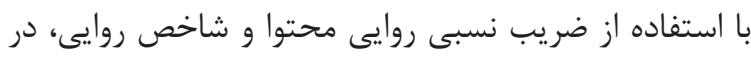

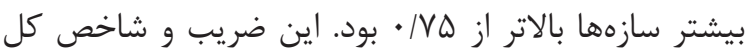

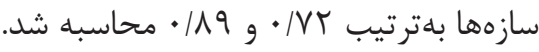

افزون بر آنجه بيان شد، محاسبه ثبات درونى سازهناى

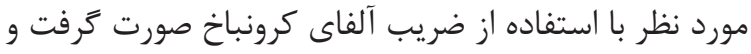

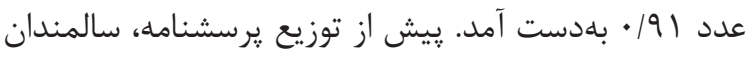

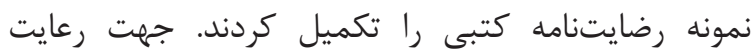

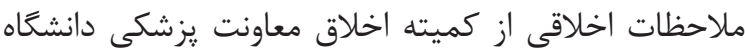
آزاد اسلامى مجوز اخذ شد (IR.IAU.REC.1395.13V).
از يزشكان در حال كشف راهكارهاى جديد مقابله با بيمارى

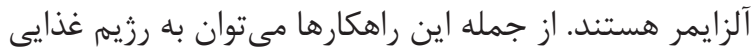

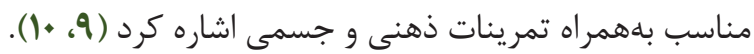

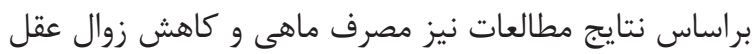

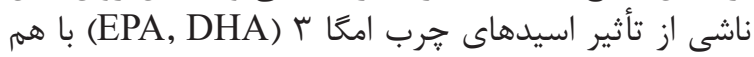

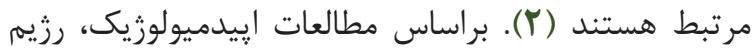

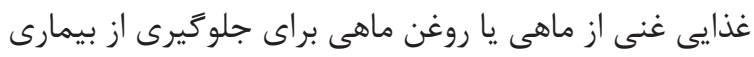

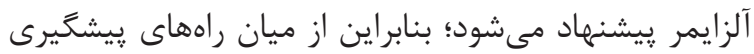

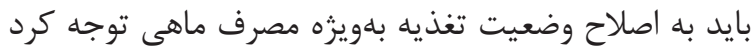

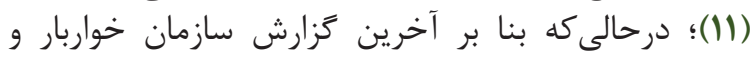

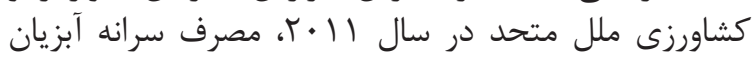

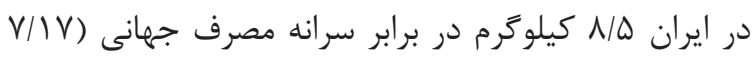

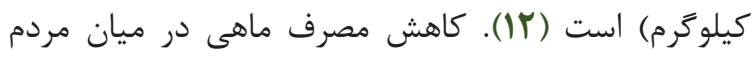

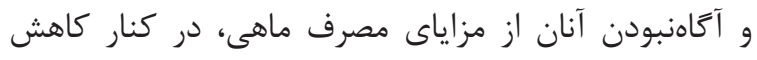

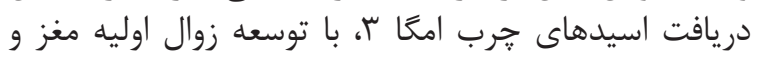

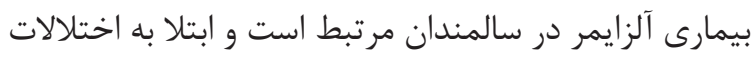

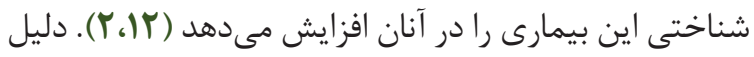

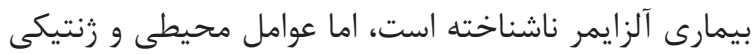

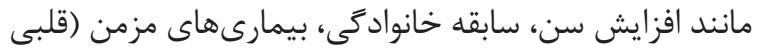

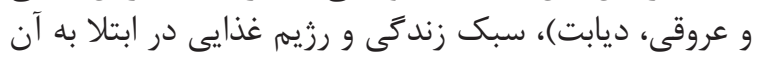

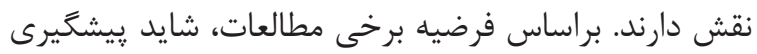

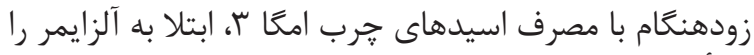

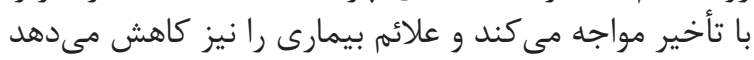

در ايران مطالعات محدودى مرتبط با عوامل تعيين كننده

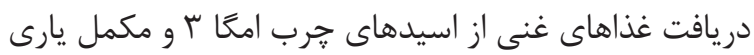

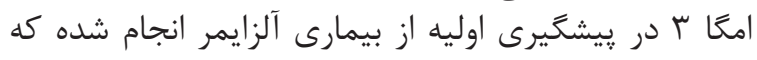

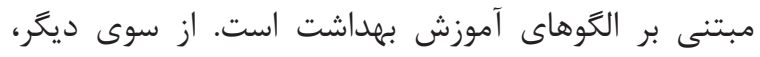

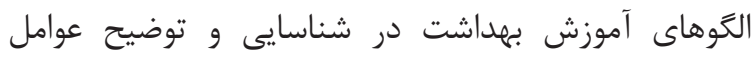

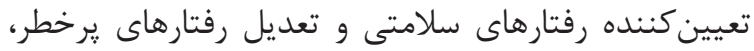

در مطالعات بسيارى اثبات شده است (1F).

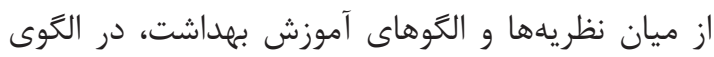

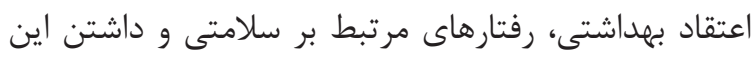

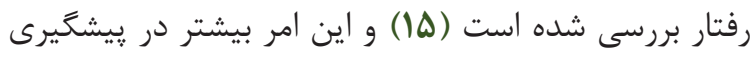

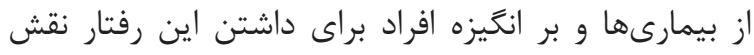

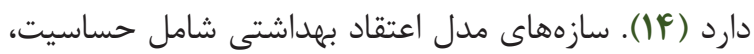

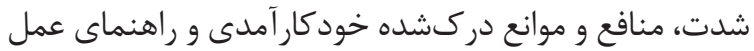

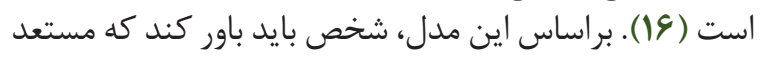

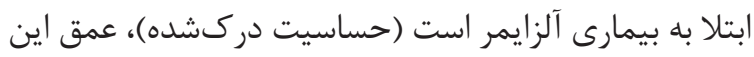

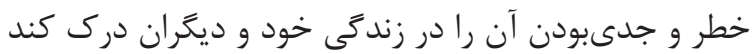

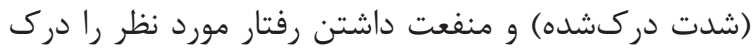

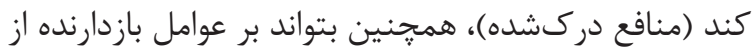

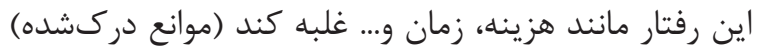

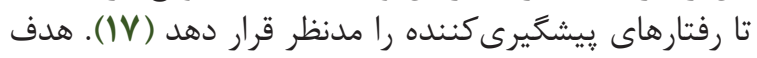

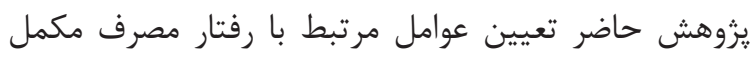

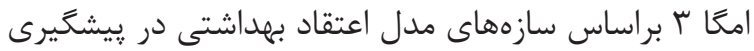

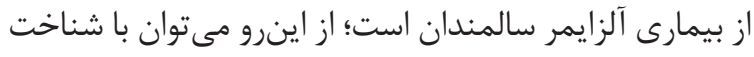

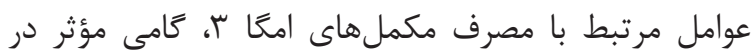


دركشده، V/T درصد نمره قابل اكتساب شدت دركشده،

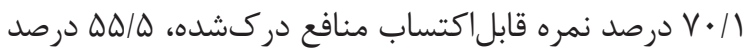

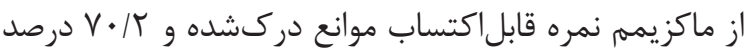

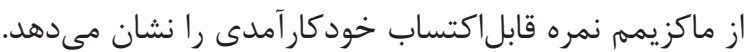
در بررسى وضعيت همبستگى ميان سازههاى الكو و رفتار

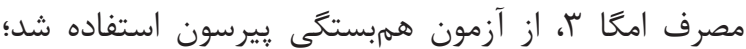

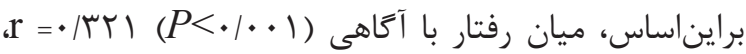

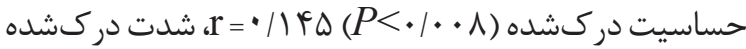

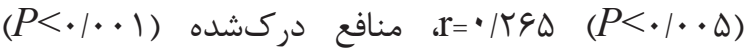

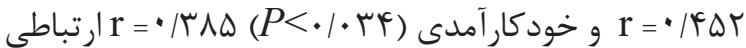

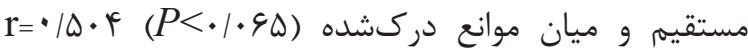
ارتباط معكوس معنادارى وجود دارد. مواع درن

براساس جدول س، سالمندان مورد مطالعه نمره متوسط

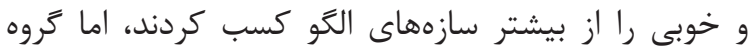
بىسوادان كمترين ميانغين را بهدست آوردند.

نتايج ديخر اين مطالعه نشان مىدهد تنها ه/ها د درصد از

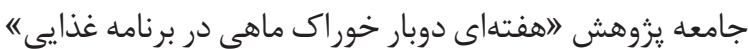

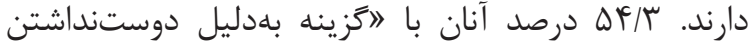

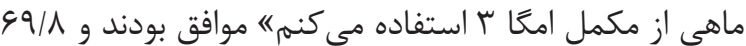

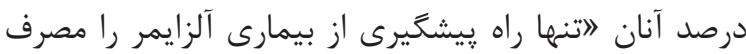

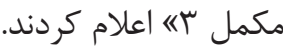

براى تحليل دادهها با استفاده از نرمافزارSPSS نسخه צا، از آزمونهاى آمار توصيفى (ميانگين و انحراف معيار)

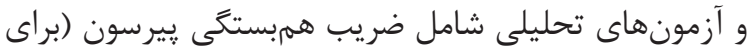

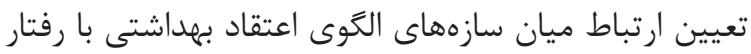

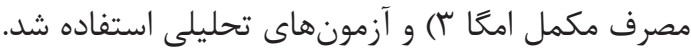

بافته ها

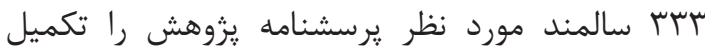

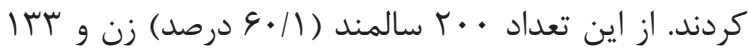

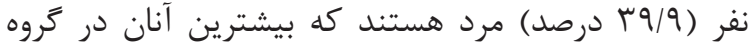

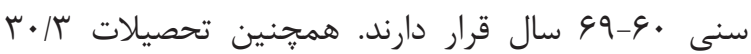

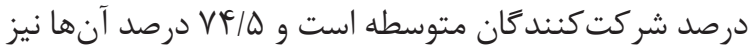

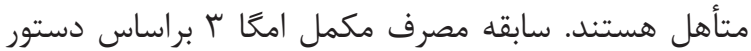

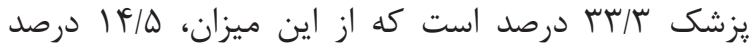
بلمنظور كاهش جربى خون،

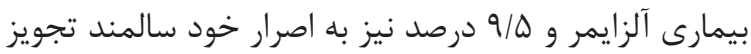

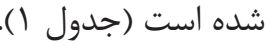

با توجه به جدول r، بالاترين ميانگين و انحراف معيار

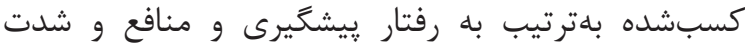

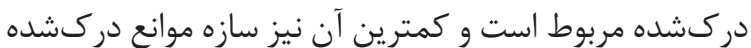

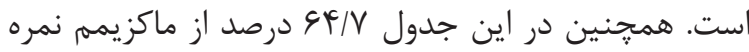

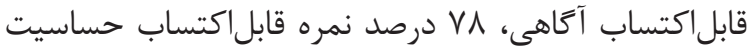

جدول شماره ا. اطلاعات جمعيتشناختى افراد مورد مطالعه

\begin{tabular}{|c|c|c|c|}
\hline درصد & تعداد & وضعيت & متغيرها \\
\hline $9 \cdot 11$ & $r$. & زن & \multirow{2}{*}{ جنس } \\
\hline ५ /१ & זrו & مرد & \\
\hline $9 \cdot / \pi$ & $r \cdot 1$ & $9 \cdot-99$ & \multirow{3}{*}{ سن } \\
\hline$r T / l$ & $1 \cdot V$ & $V \cdot-v q$ & \\
\hline$V / \Delta$ & $r \Delta$ & $\Lambda \cdot-19$ & \\
\hline$V F / D$ & TFA & متأهل & \multirow{4}{*}{ وضعيت تأهل } \\
\hline $\mathrm{V} / \mathrm{\Lambda}$ & re & مجرد & \\
\hline$r / \mathcal{F}$ & $\wedge$ & مطلقه & \\
\hline $10 / \mu$ & $\Delta 1$ & بيوه & \\
\hline $10 / \mu$ & $\Delta 1$ & بىسواد & \multirow{4}{*}{ سطح تحصيلات } \\
\hline TN/T & $9 f$ & ابتدايى & \\
\hline$r \cdot / \mu$ & $1 \cdot 1$ & متوسطه & \\
\hline$r \& / 1$ & $\Lambda \vee$ & دانشخاهى & \\
\hline r/r & $1 \cdot 1$ & تجويز يزشك & \multirow{2}{*}{ سابقه مصرف مكمل امعا r } \\
\hline $99 / V$ & TMT & بدون تجويز & \\
\hline
\end{tabular}




\begin{tabular}{|c|c|c|c|c|}
\hline \multicolumn{5}{|c|}{ 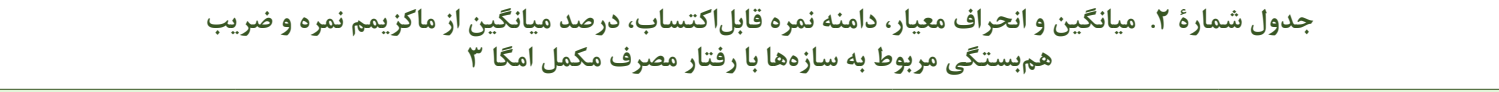 } \\
\hline ضريب همبستكى & درصد ميانكين از ماكزيمه نمره & دامنه نمره قابل اكتساب & ميانكين و انحراف معيار & 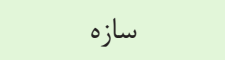 \\
\hline $\begin{array}{l}\mathrm{r}=\cdot / \mu r \mid \\
P<\cdot / \cdot \cdot 1\end{array}$ & $G 4 / V$ & $\mid \Lambda-\Delta F$ & $r \Delta / \varphi \pm r / r$ & آكاهى \\
\hline $\begin{array}{l}\mathrm{r}=\cdot / / \uparrow \Delta \\
P<\cdot / \cdot \wedge\end{array}$ & $\vee \wedge$ & $\Delta-r \Delta$ & $r V / F \pm r / Q$ & حساسيت دركشده \\
\hline $\begin{array}{l}\mathrm{r}=\cdot / r \& \Delta \\
P<\cdot / \cdot \bullet \Delta\end{array}$ & $V \Psi / T$ & $11-\Delta \Delta$ & $f \cdot / f \pm f / l$ & شدت دركشده \\
\hline $\begin{array}{l}\mathrm{r}=\cdot / \mathscr{A} \Delta \mathrm{r} \\
P<\cdot / \cdot \boldsymbol{P}\end{array}$ & $v \cdot / 1$ & $1 T-9$. & $R T / T \pm T / T \Delta$ & منافع دركشده \\
\hline $\begin{array}{l}\mathrm{r}=\cdot / \Delta \cdot \varphi \\
P<\cdot / \cdot 9 \Delta\end{array}$ & $\Delta \Delta / \Delta$ & $V-r \Delta$ & $|V / F V \pm r /| Q$ & موانع دركشده \\
\hline $\begin{array}{l}\mathrm{r}=\cdot / r \wedge \Delta \\
P<\cdot / \cdot r \digamma\end{array}$ & $V \cdot / r$ & $\Lambda-\uparrow \cdot$ & $r N / r \pm 1 / Q$ & خود كارآ آمدى \\
\hline $\begin{array}{l}\mathrm{r}=\cdot / \mu 11 \\
P<\cdot / \cdot 1\end{array}$ & $\Delta \Delta / r$ & $1 \cdot-\Delta \cdot$ & $F / / G \pm F / Q$ & رفتار ييشخيرى \\
\hline
\end{tabular}

جدول شمارة ب. توزيع فراوانى وضعيت سازههاى مدل اعتقاد بهداشتى در ميان سالمندان شركت كننده در مطالعه

\begin{tabular}{|c|c|c|c|}
\hline درصد & تعداد & دامنه نمره & سازههاى مدل \\
\hline $1 r / \Delta$ & et & ضعيف & حساسيت دركشده \\
\hline$(1 / \mathrm{V}$ & (149 & متوسط & \\
\hline$r \Delta / \Lambda$ & IDT & خوب & \\
\hline$V / \Lambda$ & rq & ضعيف & شدت دركشده \\
\hline Tr/l & w & متوسط & \\
\hline $99 / 1$ & r. & خوب & \\
\hline $\mid r / r$ & i) & ضعيف & منافع دركشده \\
\hline ( & 114 & متوسط & \\
\hline$\Delta F / 1$ & $M$ & خوب & \\
\hline $9 / \mu$ & m & ضعيف & موانع دركشده \\
\hline$r \Delta / \varepsilon$ & IQT & متوسط & \\
\hline 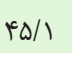 & 10 & خوب & \\
\hline $\mid \varepsilon / \Lambda$ & $\Delta F$ & ضعيف & خودكار آمدى \\
\hline$p 4 / 1$ & Ifr & متوسط & \\
\hline rq/1 & $\mu$. & خوب & \\
\hline $11 / 1$ & rq & ضعيف & رفتار \\
\hline$F V / f$ & 101 & متوسط & \\
\hline$c \cdot 1 /$ & عسו & خوب & \\
\hline N/S & gr & ضعيف & آَاهاهى \\
\hline rV & 9. & متوسط & \\
\hline$\Delta F / F$ & $M$. & خوب & \\
\hline
\end{tabular}


مى آيند (1) (1). بيش از نيمى از نمونههاى مورد مطالعه با

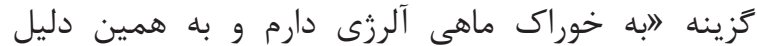

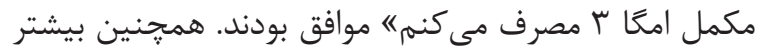

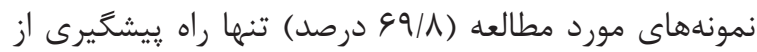

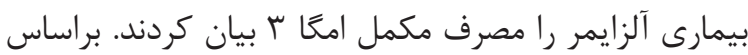

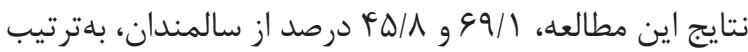

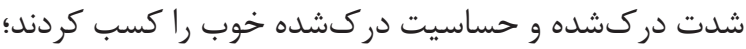

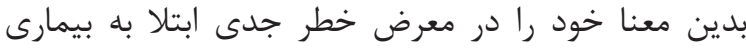

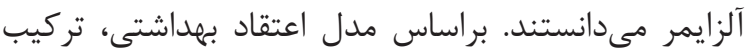

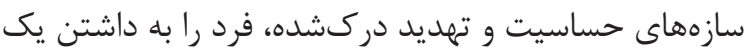

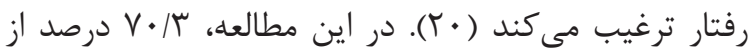

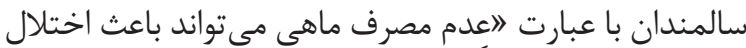

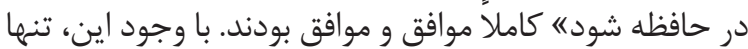

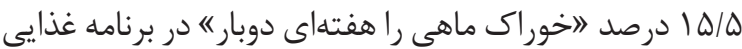

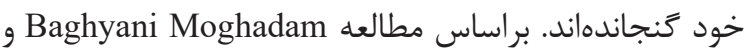

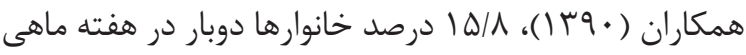

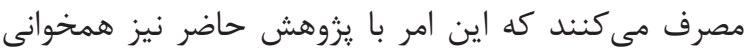

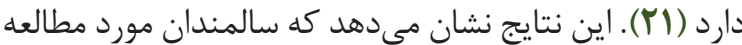

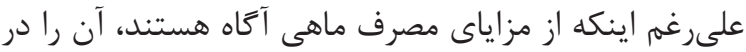

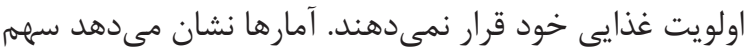

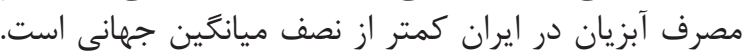

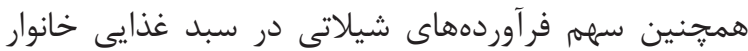

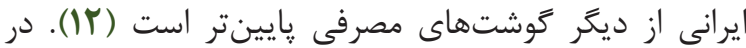

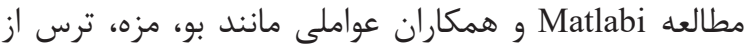

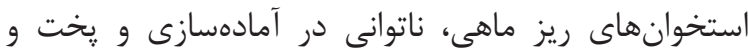

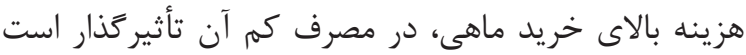

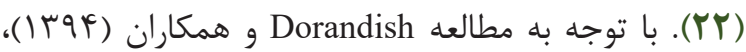

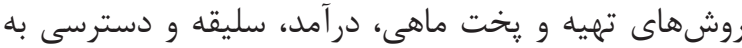

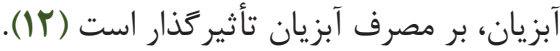

بايد توجه داشت كه نقش سازه خودكارآمدى درك مشده

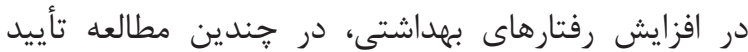

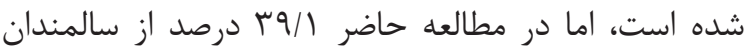

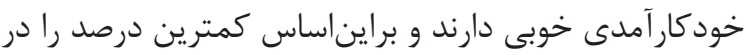

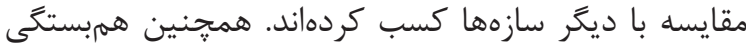

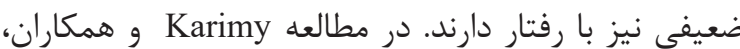

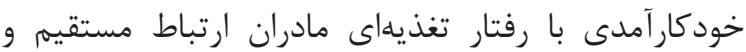

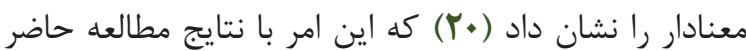

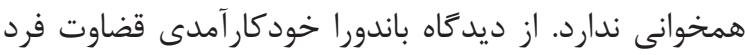

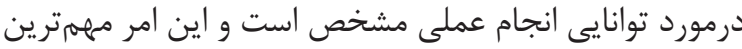
بيششرط تغيير رفتار بهشمار مى آيد (Y) (Y).

با توجه به نتايج مطالعه حاضر، همبستكى معنى دمنادارى

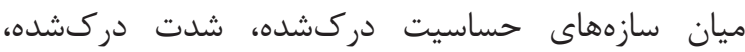

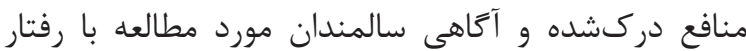

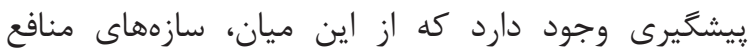

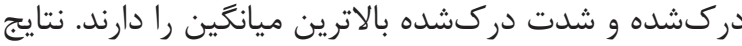

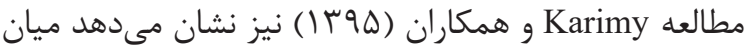

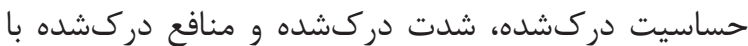

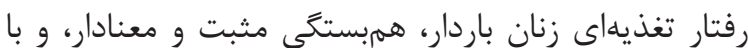

راز ميان متغيرهاى جمعيتشناختى، سطح تحصيلات

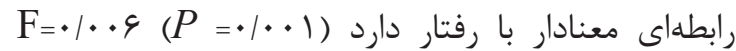

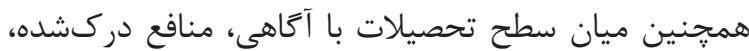

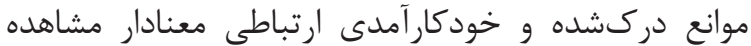

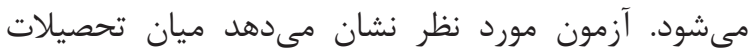

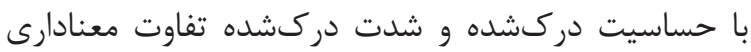

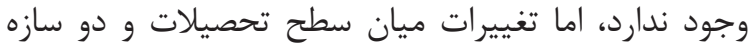

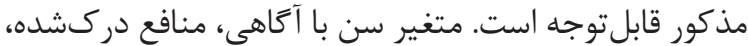

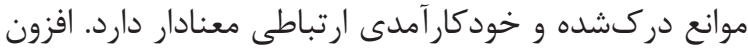

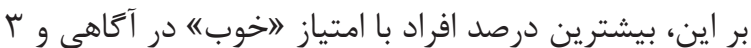

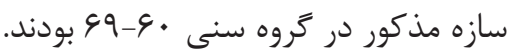

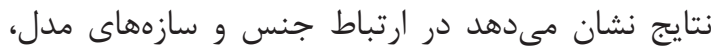

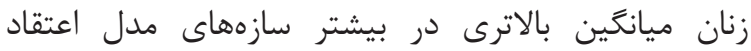

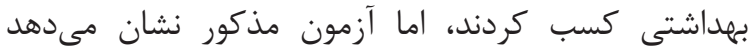

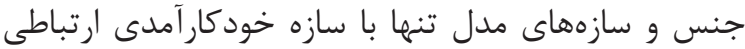

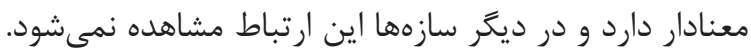

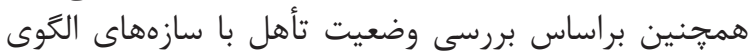

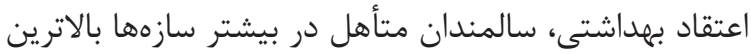

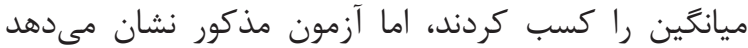

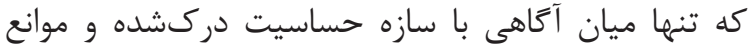
دركشده تفاوت معنادارى وجود دارد.

\section{بحث}

يزوهش حاضر براساس مطالعات بريشين انجام شد كه

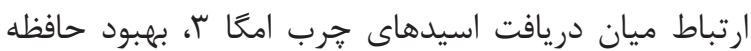

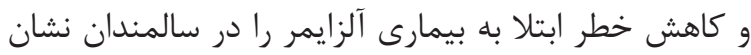

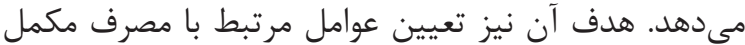

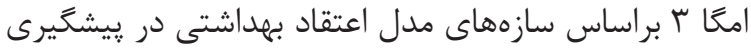

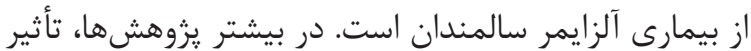

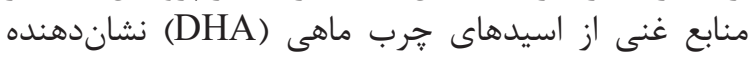

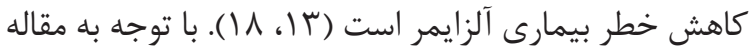
Jicha

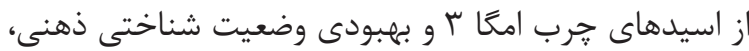

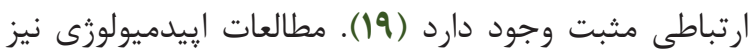

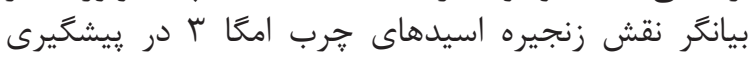

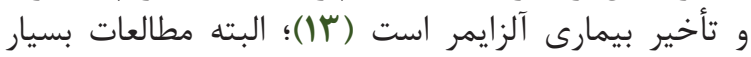

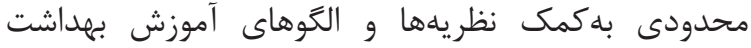

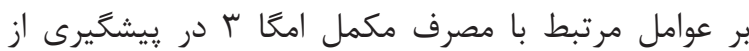

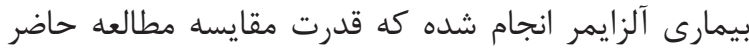

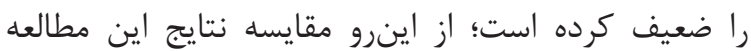

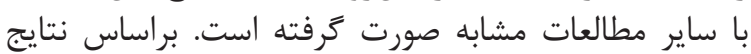

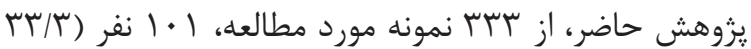

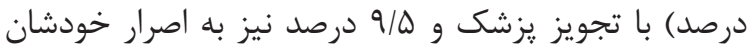

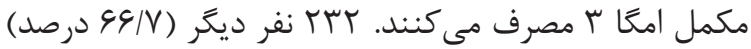

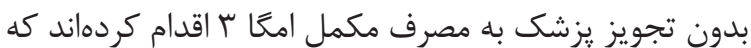

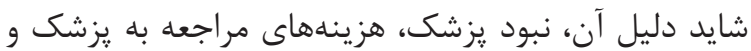

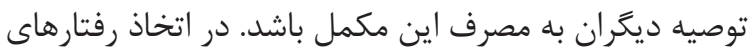

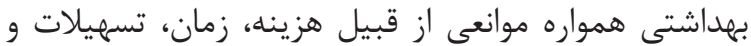

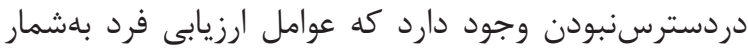




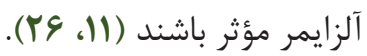

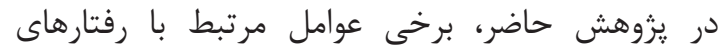

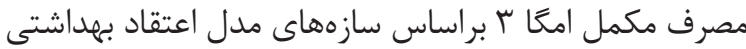

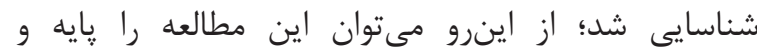

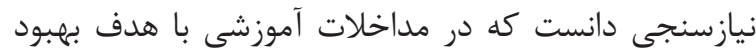

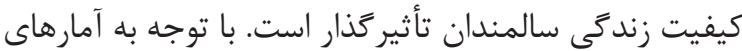

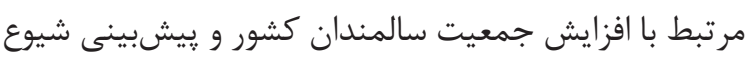

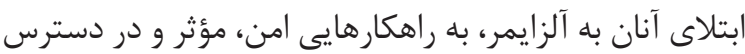

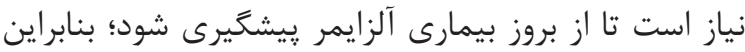

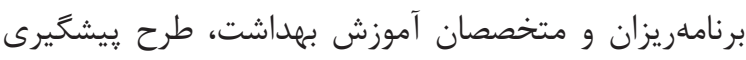

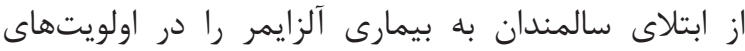

$$
\begin{aligned}
& \text { برنامهريزى آموزشى و يزوهشى كشور قرار مى دهند. } \\
& \text { سياسگزارى }
\end{aligned}
$$

مقاله حاضر حاصل طرح يزوهشى مصوب دانشعاه آزاد

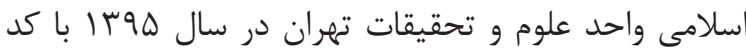
IR.IAU.REC.1395.13V

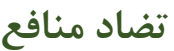

سهمم تمامى نويسندكان در اين مطالعه يكسان است و

$$
\text { هيجَّونه تضاد منافعى وجود ندارد. }
$$

\section{References}

1. Goharinezhad S, Maleki M, Baradaran H, Ravaghi H. Futures of elderly care in Iran: A protocol with scenario approach. Med J Islam Repub Iran. 2016; 30: 416. PMID: 28210581

2. Newton W, Mc Manus A. Consumption of fish and Alzheimer's disease. J Nutr Health Aging. 2011; 15(7):551-4552. PMID: 21808933.

3. Tajvar M, Arab M, Montazeri A. Determinants of health-related quality of life in elderly in Tehran, Iran. BMC Public Health20088:323.

4. Langa K. Is the risk of Alzheimer's disease and dementia declining? Alzheimer's Research \& Therapy. 2015; 7:34. https://doi.org/10.1186/ s13195-015-0118-1

5. Conn V, Stuart G, Laraia T. Families as resources, caregivers and collaborative. Principles and practice of psychiatric nursing. 8th ed. USA: Mosby; 2009.

6. Sabayan B, Bonneux L. Dementia in Iran: how soon it becomes late! Arch Iran Med 2011; 14: 290-291.

7. Maghsoudnia S. Primary health care for old adults in IRAN. University of social welfare and rehabilitation sciences publication. 1st Ed. 1385:
موانع دركشده همبستخى معكوس و معنادارى وجود دارد

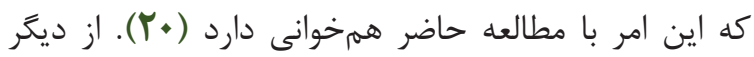

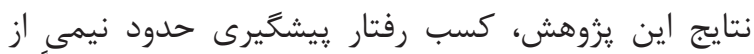

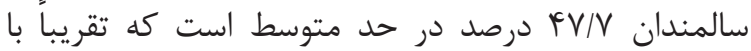

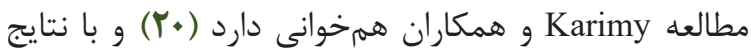

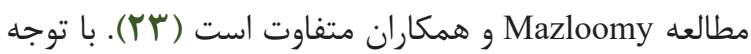

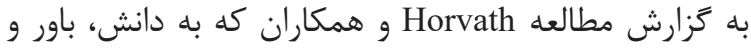

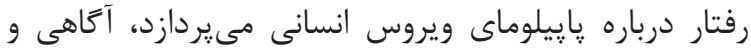

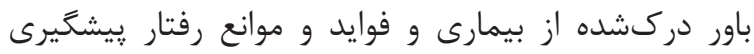

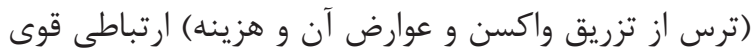

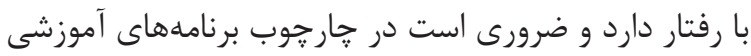
با جديت مديريت شود (YF). نتيجهَ تيرى

در حال حاضر جمعيت جهان در حال ييرشدن است؛ ازئ

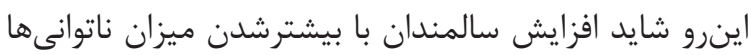

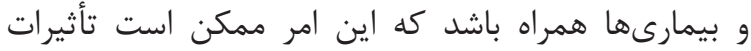

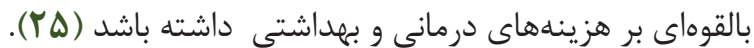

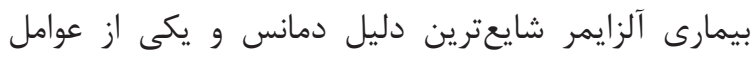

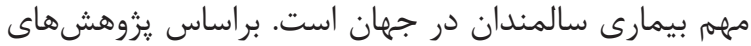

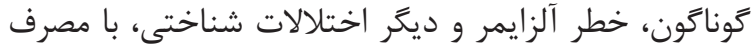

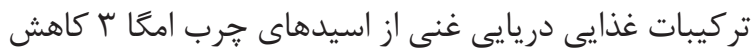

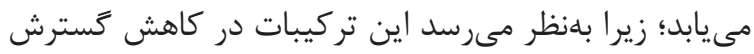

\section{7- 125.}

8. S Roriz-Filho J, Sá-Roriz T, Rosset I, Camozzato A, Santos A, Chaves M, et al. (Pre)diabetes, brain aging, and cognition.Bioch him Biophys Acta. 2009 May; 1792(5):432-43.

9. Maria van Gelder B, Tijhuis M, Kalmijn S, Kromhout D. Fish consumption, n-3 fatty acids, and subsequent 5 -y cognitive decline in elderly men: the Zutphen Elderly Study. Am J Clin Nutr. 2007; 85:1142-7. https://doi.org/10.1093/ ajcn/85.4.1142

10. Cummings J. Alzheimer's disease. N Engl J Med 2004; 351: 56-67. https://doi.org/10.1056/NEJMra040223

11. Bagheri Dizaj M, lavi Naeini A, Yekaninejad M, Mirzaei K. The relationship between dietary patterns and mild cognitive impairment in retired elderly women in Tehran. The Medical Journal of Mashhad University of Medical Sciences. 2007; 10:502-690

12. Dorandish A, Hosinzadeh M, Nematollahi Z, Evaluation of effective factors in seafood consumption in Mashad, Comparison of A homogeneous double-hurdle model. Agricultural Economics journal. 2016; 9(4):197-919

13. Thomas J, Thomas CJ, Radcliffe J, Itsiopoulos C. 
Omega-3 Fatty Acids in Early Prevention of Inflammatory Neurodegenerative Disease: A Focus on Alzheimer's disease. Biomed Res Int. 2015. https://doi.org/10.1155/2015/172801

14. Glanz K, Rimer BK, Viswanath K, editors. Health behavior and health education: theory, research, and practice. John Wiley \& Sons; 2008: 8- 30.

15. Ghaffari M, Sharifirad G, Akbari Z, Khorsandi M, Hassanzadeh A. Health Belief Model-Based Education \& Reduction of Cesarean among Pregnant Women: An Interventional Study. Journal of Health System Research. 2011; 7(2): 201-207.

16. Sharma M, Romas J. Theoretical Foundations of Health Education and Health Promotion 2nd Edition. Jones \& Bartlett. 2012: 74-90.

17. Nejad Dadgar N, Shojaeizadeh D, Tol A, Hossaini M, Amani F. Assessing the Effect of Blended Educational Program Based on Health Belief Model on Adopting Preventive Behaviors in Alzheimer among Elders under Covering Ardebil Health Care Setting. Journal of Health System Research. 2014; 10(2): 386-394.

18. Lim G, Calon F, Morihara T, Yang F, Teter B, Ubeda O, et al. A Diet Enriched with the Omega-3 Fatty Acid Docosahexaenoic Acid Reduces Amyloid Burden in an Aged Alzheimer Mouse Model. Journal of Neuroscience. 2005; 25 (12): 3032-3040. https://doi.org/10.1523/JNEUROSCI.4225-04.2005

19. Jicha G, Markesbery W. Omega-3 fatty acids: potential role in the management of early Alzheimer's disease. Clin Interv Aging. 2010; 5: 45-61. https://doi.org/10.2147/CIA.S5231

20. Karimy M, Taher M, Azarpira H. Measure health belief model construct about nutritional practices of pregnant women in Saveh University of Medical Sciences. Sci J Hamadan Nurs Midwifery Fac. 2016; 24 (3):167-173. https://doi.org/10.21859/ nmj-24034
21. Baghyani Moghadam M, Eivazi S. Investigation of Factors Related to Lack of Using Fish at The Recommended Amount by WHO in Families of Javanrood(western Iran) According to Model Goal- directed Behavior (MGB). Scientific Journal of Ilam University of Medical Sciences. 2011; 19(1):39-45.

22. Matlabi M, Sharifirad G, Mostavafi F, Mohebi S, Azadbakht L. Factors Affecting Fish Consumption Based on Structures of Health Education. Journal of Health System Research. 2012; 8(4): 523-536.

23. Mazloomy S, Zahed S, Emami M, Barekatain M, Shahnazi H, Mahaki B, A study on the evaluation of relationship between the predisposing factors and practice and demographic variables in caregivers of patients with Alzheimer's diseases. Journal of Isfahan Medical School. 2015; 33(329): 429-441.

24. Horvath, Jaqueline D.C. Human papillomavirus knowledge, beliefs, and behaviors: questionnaire adaptation. European Journal of Obstetrics \&Gynecology and Reproductive Biology.2016; 230: 103-108. https://doi.org/10.1016/j. ejogrb.2018.09.023

25. Maghsoudi A, Mohammadi Bavariani F, Salim S, Nejad-Haghighi E, Arabi H, Foruzanfar Z. The association between health promoting behaviors and quality of life in the elderly people of Ewaz, Navid No. 2016; 19(62): 16-23

26. Asadi Shavaki M, salehi L. Knowledge, belief and practice among elderly about osteoporosis based on Health Belief Model in Karaj city in 2014. Sci J Hamadan Nurs Midwifery Fac. 2016; 24 (2):86-97 